\section{Des dispositifs d'intégration clinique pour les situations complexes : aide fonctionnelle et défi normatif}

\section{Clinical integration programs for complex situations: Functional support and normative challenge}

La multimorbidité touche près de $25 \%$ de la population dans nos régions [1]. Elle entraîne une augmentation des besoins de soins et de médicaments. Malgré une perte d'autonomie fonctionnelle ou cognitive, beaucoup de malades souhaitent un maintien au domicile [2].

Leur état de santé nécessite alors la présence de plusieurs intervenants de disciplines différentes [3]. La situation du patient, déjà complexe, peut encore être aggravée par des difficultés psychosociales (isolement, précarité, etc.).

Pour répondre de façon efficiente à ces situations, les gouvernances cherchent à apporter des moyens favorisant le décloisonnement des services d'aide et de soins. La Belgique, la France et la Suisse ont mis en place des structures dont la mission est de stimuler les collaborations entre les acteurs d'aide et de soins. Cette volonté d'intégration entre les différents niveaux de soins et les services psychosociaux a pour ambition de proposer une offre de services cohérente, accessible et adaptée au patient à son domicile [4-6].

Afin de soutenir ces structures dans leur mission, chaque pays propose un moment commun de réflexion qui permet aux

TABLEAU I

Description des principales caractéristiques des CM, PPS et PSP [4-6]

\begin{tabular}{|c|c|c|c|}
\hline & $\begin{array}{l}\text { Concertation multidisciplinaire } \\
\qquad \text { (CM) }\end{array}$ & Plan personnalisé de santé (PPS) & Plan de soins partagé (PSP) \\
\hline Pays & Belgique & France & Suisse \\
\hline objectifs & $\begin{array}{c}\text { Les intervenants de première ligne } \\
\text { prennent en charge, dans son } \\
\text { milieu de vie, le patient en perte } \\
\text { d'autonomie, en favorisant la } \\
\text { qualité des soins }\end{array}$ & $\begin{array}{l}\text { Les acteurs de proximité coopèrent } \\
\text { de manière formalisée et non } \\
\text { hiérarchique, pour la personne de } \\
\text { plus de } 75 \text { ans en situation de } \\
\text { fragilité et/ou atteinte d'une (ou } \\
\text { plusieurs) maladie(s) chronique(s), } \\
\text { souhaitant être soignée dans son } \\
\text { milieu de vie }\end{array}$ & $\begin{array}{l}\text { Les participants contribuent de } \\
\text { manière non hiérarchique } \\
\text { à l'identification et l'analyse des } \\
\text { problématiques biopsychosociales, } \\
\text { chez le patient complexe ayant } \\
\text { souvent plusieurs maladies } \\
\text { chroniques et des problématiques } \\
\text { psychosociales, afin d'en } \\
\text { comprendre les causes et les effets }\end{array}$ \\
\hline $\begin{array}{l}\text { Actions concertées entre } \\
\text { les différents intervenants } \\
\text { d'aide et de soins }\end{array}$ & $\begin{array}{c}\text { Évaluer le degré d'autonomie du } \\
\text { patient }\end{array}$ & $\begin{array}{c}\text { Repérer les situations nécessitant } \\
\text { un PPS } \\
\text { Analyser les problèmes identifiés } \\
\text { et les attentes du patient avec le } \\
\text { souci d'une démarche holistique } \\
\text { Établir un plan d'action (soins, } \\
\text { aides et parfois éducation } \\
\text { thérapeutique) } \\
\text { Évaluer le suivi et réviser le plan } \\
\text { d'action }\end{array}$ & $\begin{array}{l}\text { Identifier et prioriser les problèmes } \\
\text { et les objectifs en prenant en } \\
\text { compte les attentes du patient et } \\
\text { de ses aidants proches } \\
\text { Décider les interventions, de } \\
\text { manière consensuelle et } \\
\text { participative } \\
\text { Répartir les tâches }\end{array}$ \\
\hline Reconnaissance financière & $\begin{array}{c}\text { Une intervention forfaitaire par an } \\
\text { au médecin traitant et à maximum } \\
\text { trois autres prestataires de soins } \\
\text { participant à la CM }\end{array}$ & $\begin{array}{l}\text { Une intervention forfaitaire par } \\
\text { patient nécessitant un PPS à se } \\
\text { répartir entre intervenants }\end{array}$ & $\begin{array}{l}\text { Remboursement potentiel du } \\
\text { temps consacré par les } \\
\text { intervenants aux séances } \\
\text { interprofessionnelles }\end{array}$ \\
\hline Conditions d'octroi & $\begin{array}{l}\text { Consentement du patient } \\
\text { Minimum de trois prestataires de } \\
\text { disciplines différentes } \\
\text { Renvoyer, à l'assurance, le } \\
\text { formulaire complété et signé par } \\
\text { les prestataires présents }\end{array}$ & $\begin{array}{c}\text { Consentement du patient } \\
\text { Identifier une situation qui rend } \\
\text { utile un travail formalisé entre } \\
\text { acteurs de proximité à l'aide d'une } \\
\text { grille d'évaluation }\end{array}$ & $\begin{array}{l}\text { Consentement du patient } \\
\text { Identifier le patient selon des } \\
\text { critères de complexité et des } \\
\text { bénéfices potentiels en cas de } \\
\text { prise en charge } \\
\text { interprofessionnelle }\end{array}$ \\
\hline
\end{tabular}


professionnels de s'accorder sur les objectifs et les rôles de chacun dans le respect des volontés du patient et de ses aidants proches.

Ces trois dispositifs interdisciplinaires, dont le but ultime est d'améliorer la qualité des soins, sont présentés dans le tableau I. Le cadre de référence de Valentijn [7] montre que l'intégration relève de déterminants fonctionnels (l'information, I'organisation, les finances) et normatifs (valeurs, missions). Ces déterminants sont présents aux différents niveaux du système de santé : clinique ou micro (intégration autour du patient), méso (intégration entre professionnels et/ou entre organisations) et macro (intégration au niveau du système de santé). Chaque niveau dépend des autres, en termes d'intégration.

Les premières évaluations de ces trois dispositifs [8-10] montrent qu'ils répondent bien aux besoins d'intégration clinique ainsi qu'aux souhaits du patient et des professionnels. Ils permettent d'assurer une approche holistique centrée sur le patient, de définir des objectifs communs et d'aboutir à un plan de soins négocié où les rôles sont clarifiés. La perception de la qualité des soins par les soignants, la satisfaction des patients et des intervenants est augmentée.

Pourtant, malgré leurs points forts, ces dispositifs tardent à prendre leur place. Les principaux obstacles sont le manque de temps professionnel pour ce travail de concertation, la lourdeur administrative des dispositifs, la disponibilité variable des intervenants des différentes disciplines et l'inégalité de rémunération entre les professionnels.

Parmi les pistes d'amélioration, les premières sont fonctionnelles: les intervenants pointent l'importance d'une circulation fiable de l'information. L'espoir d'un système informatisé et sécurisé des données est dans tous les esprits. Les professionnels évoquent aussi la nécessité d'une fonction de coordination pour gérer les tâches et horaires de chacun.

De plus, le travail d'équipe requiert, de la part des professionnels, une attitude collaborative. Faire connaissance et reconnaître les compétences de chacun, se respecter, avoir confiance et partager une vision et des objectifs communs sont autant de déterminants normatifs influençant la bonne collaboration interdisciplinaire. Proposer des formations interdisciplinaires dans le cursus de base et en formation continue et encourager le travail en équipe pluridisciplinaire permettrait ainsi de développer progressivement une culture interdisciplinaire. Une transformation en profondeur des attitudes professionnelles est nécessaire et prendra du temps.

\section{Conclusion}

La complexité des situations vécues à domicile nécessite un décloisonnement des professions de la santé. Les professionnels n'ont pas été formés au travail collaboratif, pourtant essentiel aux besoins d'intégration. Pour espérer une propagation d'une culture pluridisciplinaire en santé, les pouvoirs publics doivent poursuivre et renforcer leur soutien aux professionnels.
Encourager l'intelligence collective coûte du temps et de l'argent. C'est un investissement nécessaire pour les nouveaux défis de nos systèmes de santé.

Remerciements : aucun

Sources de financements : aucun.

Déclaration de liens d'intérêts : les auteurs déclarent ne pas avoir de liens d'intérêts.

\section{Références}

[1] Barnett K, Mercer SW, Norbury M, Watt G, Wyke S, Guthrie B. Epidemiology of multimorbidity and implications for health care, research, and medical education: a cross-sectional study. Lancet 2012; 380:37-43

[2] European Commission. 2010: independent living for the ageing society. Luxembourg: Office for Official Publications of the European Communities; 2006 [ISBN 92-79-02333-0].

[3] Tarricone R, Tsouros AD. Home care in Europe: the solid facts. Copenhagen (Denmark): WHO Regional Office for Europe; 200846 [Citée: 29/7/2011; ISBN 97892890 4281].

[4] Ministère des solidarités et de la santé. Le dispositif Paerpa. France; 2017 [Consulté le 24 mai 2017 ; disponible sur : http://social-sante.gouv.fr/ systeme-de-sante-et-medico-social/parcours-des-patients-et-des-usagers / le-parcours-sante-des-aines-paerpa/article/le-dispositif-paerpa].

[5] Promotion des Réseaux Intégrés des Soins aux Malades. Le patient complexe et sa prise en charge ambulatoire, Suisse; 2015. [Consulté le 24 mai 2017 ; disponible sur : http://www.prism-ge.ch].

[6] Guide projets-pilotes soins intégrés en faveur des malades chroniques; 2016 [Consulté le 24 mai 2017 ; disponible sur : http://www.integreo.be/ sites/default/files/public/content/guidepp.pdf].

[7] Valentijn PP, Schepman SM, Opheij W, Bruijnzeels MA. Understanding integrated care: a comprehensive conceptual framework based on the integrative functions of primary care. Int J Integr Care 2013;13:e010.

[8] Haute Autorité de Santé (HAS). Mise en œuvre du PPS PAERPA : bilan à 6 mois; 2015 [Consulté le 13 juillet 2016 ; disponible sur : http ://www. hassante.fr/portail/upload/docs/application/pdf/2015 12/bilan_a_6_ mois_plan_personnalise_sante_vf_web.pdf].

[9] Perone N, Schusselé Filliettaz S, Budan F, Schaller $P$, Balavoine JF, Waldvogel F. Concrétiser la prise en charge interdisciplinaire ambulatoire de la complexité. Santé Publique; 2015p. 77-86.

[10] Buret L, Duchesnes C, Giet D. La concertation multidisciplinaire : intérêt, facilitateurs et freins perçus par les professionnels d'aide et de soins; 2017 [Consulté le 31 mai 2017 ; disponible sur : http://hdl.handle.net/2268/ 208050].

Laetitia Buret, Christiane Duchesnes, Didier Giet

Université de Liège, faculté de médecine, département de médecine générale, avenue Hippocrate, 13, bâtiment B23, 4000 Liège, Belgique

Correspondance : Laetitia Buret, université de Liège, faculté de médecine, département de médecine générale, avenue Hippocrate, 13, bâtiment B23, 4000 Liège, Belgique Laetitia.buret@ulg.ac.be

Reçu le 14 septembre 2016 Accepté le 2 août 2017 Disponible sur internet le 14 septembre 2017

http://dx.doi.org/10.1016/j.lpm.2017.08.001

(c) 2017 Elsevier Masson SAS. Tous droits réservés. 